\title{
The Southern African Large Telescope project
}

\author{
David A. H. Buckley ${ }^{1,2}$, Philip A. Charles ${ }^{2}$, Kenneth H. Nordsieck ${ }^{3}$ \\ and Darragh O'Donoghue ${ }^{2}$ \\ ${ }^{1}$ SALT Foundation, PO Box 9 Observatory 7935, South Africa \\ email: dibnob@saao.ac.za \\ ${ }^{2}$ South African Astronomical Observatory \\ ${ }^{3}$ Space Astronomy Lab, University of Wisconsin-Madison
}

\begin{abstract}
The recently completed Southern African Large Telescope (SALT) is a low cost, innovative, $10 \mathrm{~m}$ class optical telescope, which began limited scientific operations in August 2005, just 5 years after ground-breaking. This paper describes the design and construction of SALT, including the first-light instruments, SALTICAM and the Robert Stobie Spectrograph (RSS). A rigorous systems engineering approach has ensured that SALT was built to specification, on budget, close to the original schedule and using a relatively small project team. The design trade-offs, which include an active spherical primary mirror array and a fixed altitude telescope with a prime focus tracker, although restrictive in comparison to conventional telescopes, have resulted in an affordable $10 \mathrm{~m}$ class telescope for South Africa and its ten partners. Coupled with an initial set of two seeing-limited instruments that concentrate on the UV-visible region $(320-900 \mathrm{~nm})$ and featuring some niche observational capabilities, SALT will have an ability to conduct some unique science. This includes high time resolution studies, for which some initial results have already been obtained. Many of the versatile modes available with the RSS - which is currently being commissioned - are unique and provide unparallelled opportunities for imaging polarimetry and spectropolarimetry. Likewise, Multi-Object Spectroscopy (with slit masks) and imaging spectroscopy with the RSS, the latter using Fabry-Perot étalons and interference filters, will extend the multiplex advantage over resolutions from 300 to 9000 and fields of view of 2 to 8 arcminutes. Future instrumentation plans include an extremely stable, fibre-fed, high resolution échelle spectrograph and a near-IR (to between 1.5 to $1.7 \mu \mathrm{m}$ ) extension to the RSS. Future development possibilities include phasing the primary mirror and AO. Finally, extrapolations of the SALT/HET designs to ELT proportions remain viable and are surely more affordable than conventional, fully steerable, designs.
\end{abstract}

Keywords. telescopes - instrumentation: detectors, photometers, polarimeters, spectrographs — stars: individual (SDSS015543+002807).

\section{Introduction and background}

South Africa, together with ten international partners from five countries, has just completed and inaugurated - on 10 Nov 2005 - the Southern African Large Telescope (SALT), at a cost of $\sim$ US $\$ 19.7 \mathrm{M}$. SALT is based on the innovative design of the HobbyEberly Telescope (HET), at McDonald Observatory (Texas), which began science operations in October 1999. These telescopes represent a completely new design paradigm for optical/IR telescopes (Sebring et al. 1997, Ramsey et al. 1998, Stobie, Meiring \& Buckley 2000), being optical analogues of the Arecibo radio telescope. A segmented spherical primary mirror array of diameter $11 \mathrm{~m}$, consisting of 91 identical hexagonal segments with spherical surfaces, directs light to a 4 mirror spherical aberration corrector (SAC), 
mounted on a moving tracker at the prime focus. Significant design changes and enhancements were made to SALT (Meiring et al. 2003, Meiring \& Buckley 2004) following upon the experiences and lessons learnt with the HET in its early operations phase, and these are described in Section 5 .

SALT is owned by a Foundation, set up to fund its construction and operation, which represents a collaboration of universities and institutes from Africa, Europe, New Zealand and North America. The South African National Research Foundation is a majority shareholder with $34 \%$ of the observing shares. Other major shareholders are Dartmouth College (14\%), University of Wisconsin-Madison (14\%), Nicolaus Copernicus Astronomical Centre of the Polish Academy of Sciences (11\%) and Rutgers University of New Jersey (10\%). Other shareholders (at $\lesssim 5 \%$ level) include the Carnegie Mellon University, University of Canterbury, University of North Carolina, Göttingen University and the UK SALT Consortium representing the Universities of Lancashire, Keele, Nottingham and Southampton, the Open University and the Armagh Observatory. Participation in SALT was attractive to its partners for a number of reasons, the most notable being:

- Access to a $10 \mathrm{~m}$ class telescope

- Access to the southern hemisphere

- Affordable ownership

- Good observatory site

- Synergies with other facilities accessible by SALT partners

- Relatively inexpensive queue-scheduled service operations

- Assisting in the development of science and technology and educational opportunities in South Africa

\section{SALT history}

The first milestone for SALT occurred on 1 June 1998 when the South African Government conditionally approved the construction of SALT, once matching funds were committed. By 25 November 1999 sufficient international funding had been committed to allow the declaration of 'green light' for the construction of SALT to proceed. The SALT project team was hired by early 2000 and the next major milestone was the ground breaking ceremony, held on 1 September 2000. Many of the major components of the telescope, including the building, were completed in 2002, and the first mirrors were installed in December of that year. October 2003 saw the commencement of the first on-sky engineering tests, following the installation of the Prime Focus Tracker \& Payload the previous month. By the end of the year the first closed loop guided observations were obtained, with 18 mirror segments installed and using a surrogate spherical aberration corrector (SAC) borrowed from the HET. The SALT SAC was finally installed in July 2004 and installation of the capacitive edge sensors also began in that year. The end of 2004 saw further progress with the on-sky testing, including the guidance and focus system and general maturing of the telescope control system. The final batch of mirrors were installed in May 2005, followed by the testing of the first science instrument, SALTICAM. Commissioning observations with this instrument began in August 2005, which was followed on 1 September by the declaration of "first light", with all mirrors in place and SALTICAM fully operational - exactly five years following ground breaking.

The remainder of 2005 has been taken up with continuing engineering shakedown, testing of the edge sensor system, plus "performance verification" observations with SALTICAM. This was the first opportunity for all astronomers within the SALT partnership to obtain data. October saw the installation and beginning of commissioning of the second "first generation" instrument, the Robert Stobie Spectrograph (RSS), named in honour 


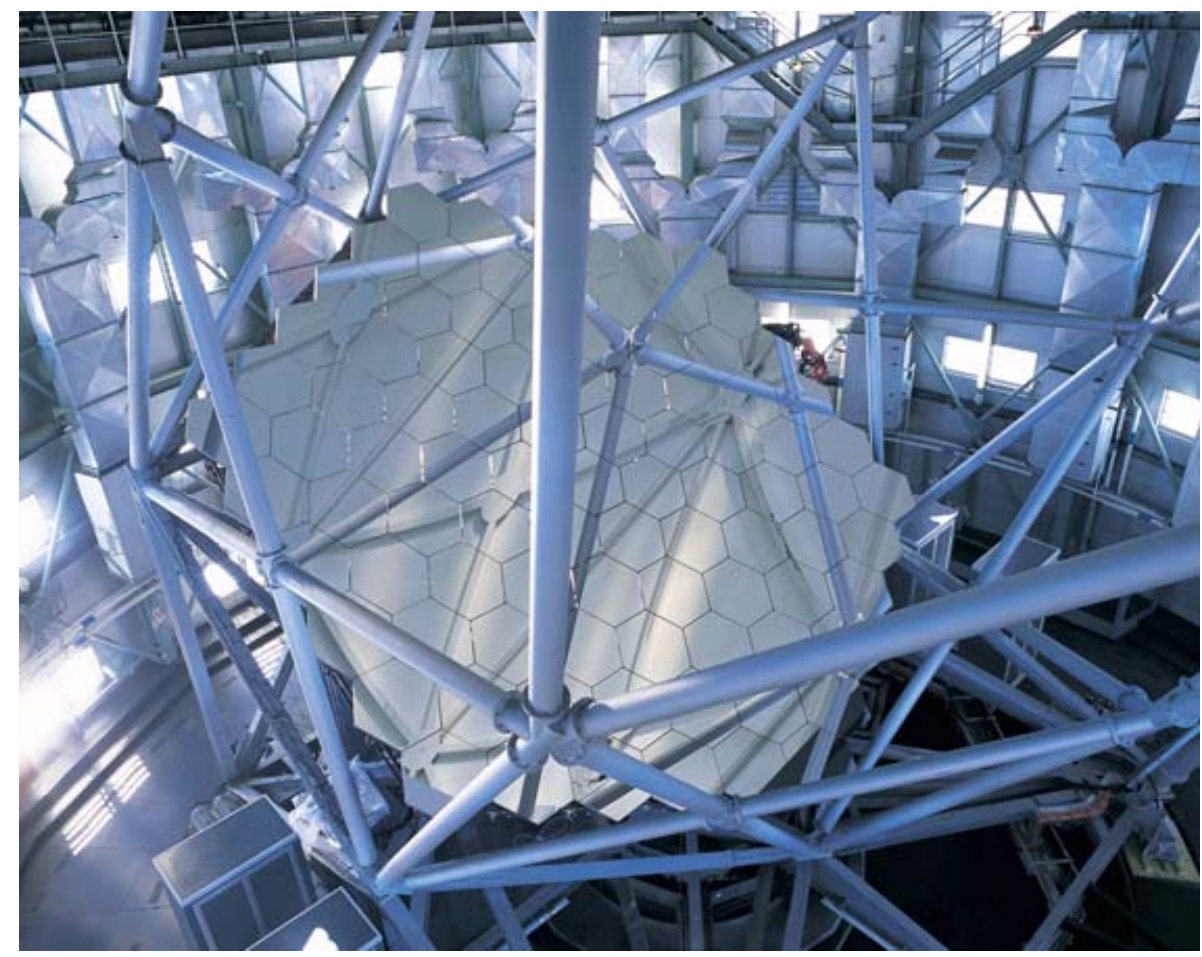

Figure 1. The SALT primary mirror array, consisting of 91 hexagonal Astrositall segments $1.2 \mathrm{~m}$ from corner-to-corner and figured, with an accuracy of $15.7 \mathrm{~nm}$ RMS, to a master sphere of $26.165 \mathrm{~m}$ radius of curvature.

of Bob Stobie, one of the instigators of SALT, its first Board Chairperson and SAAO Director, until his untimely death in May 2002. At the time of this meeting, the first science paper, based on SALTICAM data, has just been submitted, and the telescope is now routinely attempting scientific observations.

\section{SALT location and environment}

SALT was built at the existing Sutherland observing station (established in 1972) of the South African Astronomical Observatory (SAAO) situated on a plateau in the Karoo semi-desert, at an altitude of $\sim 1800 \mathrm{~m}$. Observing conditions are 'spectroscopic' or better for $\sim 75 \%$ of the nights and $\sim 50 \%$ of the nights are 'photometric.' The site exhibits little in the way of seasonal variations and is very dark being situated in a remote and unpopulated region. Conditions are often very dry, which allows for routine near infrared $(J H K)$ observations, while the median zenithal seeing (in the $V$-band, as measured by DIMMs) is $0.92 \operatorname{arcsec}(\mathrm{FWHM})$. Sutherland is also one of the most seismically quiet regions on Earth.

SALT is designed to operate optimally in the following environmental conditions:

- Over a temperature range of -5 to $20^{\circ} \mathrm{C}$,

- In steady winds up to $\sim 60 \mathrm{~km} \mathrm{~h}^{-1}\left(\sim 17 \mathrm{~m} \mathrm{~s}^{-1}\right)$, and

- Relative humidity $<97 \%$.

In addition, the telescope can continue operating, with a $20 \%$ degraded performance, for winds up to $75 \mathrm{~km} \mathrm{~h}^{-1}$ and temperatures $5^{\circ}$ lower/higher than the nominal limits (i.e. -10 to $\left.25^{\circ} \mathrm{C}\right)$. 


\section{SALT science requirements}

A set of science requirements for SALT were defined in order to meet the scientific goals of the SALT partners. These high level requirements were in turn used to define the overall system technical specification and in turn the subsystem and component specifications. This is in keeping with a standard systems engineering approach involving a structured development process, which was adopted throughout the SALT project (Swart \& Meiring 2003, Swart \& Buckley 2004).

SALT is designed to be seeing-limited and will be most competitive spectroscopically, although its imaging capability has been greatly enhanced compared to the HET due to its redesigned SAC (O'Donoghue 2000, O'Donoghue \& Swat 2001). The science field diameter is 8 arcmin and the image quality, specified in terms of enclosed energy (EE) diameter, to be $\operatorname{EE}(80)<0.9$ arcsec (i.e. $80 \%$ of the energy of the PSF falls within a 0.9 arcsec diameter circle) and $\mathrm{EE}(50)<0.6$ arcsec, where the median zenithal seeing FWHM is 0.9 arcsec. Thus SALT is designed to not significantly degrade the natural site seeing profile. While the primary mirror of SALT is not phased, due consideration was given to allow a possible upgrade for phasing in the future, e.g. by defining the appropriate specifications on the mirror actuators and edge sensors.

Other requirements for SALT included an accurate tracking capability, including field rotation, nodding and offset auto-guiding and auto-focusing. Operational efficiency was also of prime importance, requiring an ability to quickly acquire and centre objects on instrument entrance slits, apertures, fibres, etc. Many of the science drivers for SALT require an ability to operate at short wavelengths, down to the UV atmospheric cutoff at $\sim 320 \mathrm{~nm}$. This led to a requirement for high telescope throughput from 320 to $2500 \mathrm{~nm}$. This was indeed achieved by using newly developed multi-layer coatings on the SAC mirrors.

As many exciting science programs utilise the light gathering power of SALT to the limit where sky background becomes dominant, minimising scattered and stray light is important. An efficient and accurate calibration system, excellent tracking and atmospheric dispersion compensation are also key telescope requirements.

The restricted viewing window of SALT implies that as much astrophysical information should be obtained per unit time interval as possible. This implies maximising the collecting area of the telescope, minimising light losses and optimising the track trajectory to ensure maximum photon flux. The first requirement lead to the choice of an $11 \mathrm{~m}$ diameter entrance pupil following the SAC redesign (O'Donoghue 2000). Telescope efficiency, particularly minimising the time needed to acquire and guide on an object, is also a crucial factor in maximising scientific productivity.

\section{SALT design enhancements}

Although the basic design of SALT is similar to that of the Hobby-Eberly Telescope (HET), there are significant departures brought about to enhance capability and performance and take advantage of the lessons learnt with the HET. Almost every subsystem on SALT has been redesigned, resulting in expected improvements in performance. These design changes included:

- A redesigned spherical aberration corrector (SAC; O'Donoghue 2000), giving a larger field of view ( 8 arcmin diameter), improved imaging quality (EE50<0.2 arcsec) and using multi-layer protected $\mathrm{Ag} / \mathrm{Al}$ coatings on the four mirrors to enhance its sensitivity at short wavelengths (capable down to $320 \mathrm{~nm}$ ). 
- An active primary mirror alignment system, which includes re-designed flexure-based mirror supports, a precision actuation system and utilisation of capacitive edge sensors to measure primary mirror segment movement. In addition, a Shack-Hartmann wavefront system is used for initial optical alignment of all the segments.

- A facility building utilising air conditioning during the day and natural ventilation at night, with controllable louvers, and measures employed to remove all heat sources inside the telescope chamber in order to minimise dome seeing. These measures include using glycol cooling of powered subsystems, which are installed inside insulated cabinets, and forced ventilation under a false observing floor during the night.

- A greatly enhanced Prime Focus Payload with 4 focal stations, and including a sensitive science grade acquisition camera (SALTICAM), separate focus and auto-guiding cameras, a facility atmospheric dispersion compensator, a moving exit pupil baffle and calibration system for flat-fields and arcs.

- An active payload alignment system, which uses both a laser auto-collimator and a Mach-Zender distance measuring interferometer to keep it optimally aligned in tip/tilt and distance with respect to the primary mirror.

- A robust, integrated, holistic and capable Telescope Control System, based on LabVIEW, which is more easily integrated, tested, commissioned and maintained.

- A set of first-light instruments that will take advantage of the expected improvements in telescope image quality, larger science field of view ( 8 arcmin diameter) and good $\mathrm{UV} /$ blue response.

\section{SALT science drivers}

For a telescope like the SALT one has to consider how it can be competitive with more versatile, and better funded, telescopes of similar aperture. Clearly without having a phased primary mirror array and adaptive optics, SALT will never be competitive in terms of imaging capability, particularly in the IR. Despite the constraints on SALT, the design improvements, particularly the improved sensitivity at shorter wavelengths, will ensure SALT is competitive in many areas. With a smaller user base, SALT also has an advantage in terms of the amount of $10 \mathrm{~m}$ class telescope time available for a given project. In choosing the attributes for SALT and its science instruments, we have been careful to ensure that we take full advantage of SALT's competitiveness, enhanced capabilities and observational "niches" (Buckley et al. 2003). Examples where we believe this will have an impact include:

- spectroscopy (with up to $\mathrm{R} \sim 10000)$ from the atmospheric UV cutoff $(320-900 \mathrm{~nm})$, long-slit and MOS,

- all-Stokes polarimetry and spectropolarimetry (i.e. linear and circular, simultaneous if necessary),

- high-speed $(\sim 10 \mathrm{~Hz})$ time resolved imaging photometry, polarimetry, spectroscopy and spectropolarimetry,

- Fabry-Perot imaging spectroscopy $(\mathrm{R}=300-9000)$, with a unique imaging spectropolarimetric mode,

- dedicated queue scheduling, easily allowing synoptic observations over a range of timescales (days to years), and

- very stable fibre-fed high dispersion $(\mathrm{R}=70000)$ spectroscopy capable of $\mathrm{m} \mathrm{s}^{-1}$ radial velocity accuracy. 


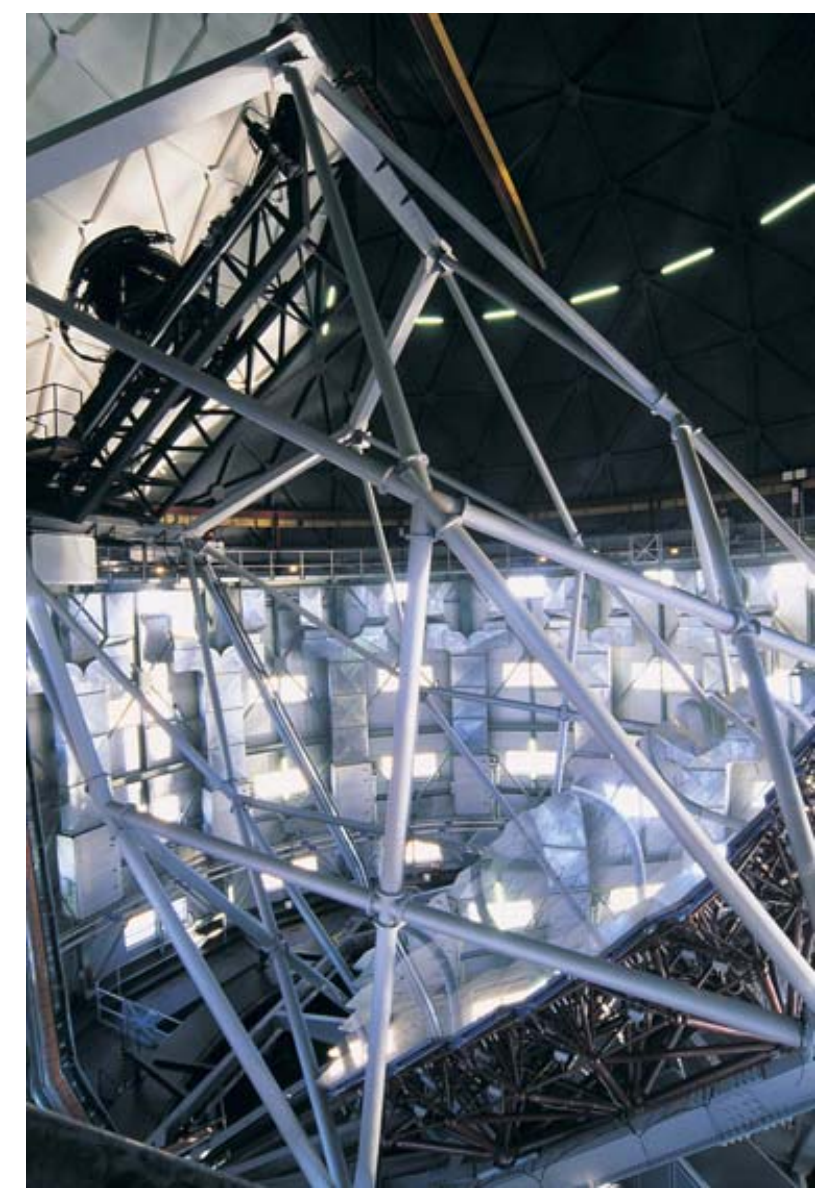

Figure 2. The SALT telescope, with the primary mirror array at the bottom right and the tracker and prime focus payload to the left, with the white dome shutter behind.

\section{SALT science instruments}

Provision was made within the SALT construction budget, through a combination of both cash and "in-kind" funding for a set of three "first-generation" science instruments (Buckley et al. 2000, 2004b). All three instruments are being built by SALT consortium members and two of these, SALTICAM and the Robert Stobie Spectrograph (RSS) were installed in 2005, while the third, the High Resolution Spectrograph (HRS), is just entering the construction phase and is therefore not expected to be completed until 2008.

The capabilities of SALT first generation instruments will initially be confined to the UV-visible domain(320-900 nm), although an upgrade path to support near IR imaging and spectroscopy is planned. The first two instruments are both mounted at prime focus thus taking advantage of the good UV/blue performance (320-450 nm) afforded by the high efficiency SAC mirror coatings. In addition, the design of the SAC affords a respectable 8 arcmin diameter science field of view. This, coupled with the active SALT primary mirror and the dynamic prime focus payload alignment system, will give SALT seeing limited imaging performance. This is fully exploited by both SALTICAM, an efficient broad-band and potentially high-speed optical imaging camera, and the RSS. HRS will be a very stable fibre fed échelle spectrograph, primarily designed for single objects, 
but with a good background subtraction ability. The HRS will be serviced by the Fiber Instrument Feed (FIF), a fiber positioning system.

\subsection{Robert Stobie Spectrograph}

Formerly known as the Prime Focus Imaging Spectrograph (PFIS), the instrument was renamed the Robert Stobie Spectrograph (RSS) just prior to its installation on SALT in October 2005. The RSS will be the initial work-horse instrument on SALT and was constructed by the University of Wisconsin-Madison, where the Principal Investigator is one of the authors (KHN). Two other SALT partners were also involved in the project: Rutgers University was responsible for the Fabry-Perot subsystem and the Invar structure, while the South African Astronomical Observatory (SAAO) built the CCD mosaic detector (O'Donoghue et al. 2004).

The RSS (Buckley et al. 2004b, Burgh et al. 2003, Kobulnicky et al. 2003) has been designed to have a range of capabilities and observing modes, each one remotely and rapidly able to be reconfigured (Kobulnicky et al. 2003). In keeping with the overall philosophy of exploiting the niche areas where SALT has a competitive edge, the instrument has several unique or rare capabilities, some afforded by various enabling technologies. The RSS capabilities include:

- The ability to observe down to the UV atmospheric cut-off, at $\sim 320 \mathrm{~nm}$. This is achieved by the judicious use of UV transmitting materials in the optical design, including $\mathrm{NaCl}(!)$, which is used in central elements of two sealed triplets. High throughput has demanded the use of efficient anti-reflection coatings, including Solgel on interior (sealed) lens surfaces.

- A fully articulating camera/detector used with Volume Phase Holographic transmission gratings (VPHGs). This, to our knowledge, is only the second spectrograph so designed to take full advantage of VPHGs (the other being the Goodman Spectrograph on SOAR).

- All Stokes mode spectropolarimetry and imaging polarimetry using either one or both $1 / 2$ and $1 / 4$ waveplate retarders and a large Wollaston beam-splitter mosaic, giving two completely off-set O- and E-images on the detector (Nordsieck et al. 2003).

- Fabry-Perot imaging spectroscopy in the range 430-860 nm using three étalons, with two sometimes in series, providing resolutions from $\mathrm{R}=320$ to $\sim 9000$.

- The use of fast frame-transfer CCDs allowing for high-speed observations in all observing modes.

The RSS wavelength coverage is from 320-900 nm, with the provision for an upgrade for a second near IR arm (to $1.7 \mu \mathrm{m}$ ) using a dichroic beam splitter. Multiple object spectroscopy (MOS) using laser cut graphite focal plane slit masks of up to 100 objects at a time will be possible. A "nod and shuffle" mode will also be employed for accurate background subtraction.

\subsubsection{RSS CCD detector}

The CCDs for the RSS (and SALTICAM) are E2V CCD 44-82 chips, each with $2 \mathrm{k} \times 4 \mathrm{k} \times 15 \mu \mathrm{m}$ pixels (O'Donoghue et al. 2004). These CCDs are thinned and backilluminated deep depletion devices coated for good UV response. They are designed to be able to be run in frame transfer mode for improved time resolution, which should allow for observations at $\sim 10 \mathrm{~Hz}$ for either high-speed spectroscopy or windowed differential photometry with insignificant readout time losses. Three of these chips were mosaiced together for RSS, i.e. an effective $4 \mathrm{k} \times 6 \mathrm{k}$ detector area, although binning by at least $2 \times 2$ will be usual for most situations $(1$ pixel $=0.13$ arcsec $)$. All the chips are cosmetic 
Grade 0 devices with low readout noise $\left(2.0-2.4 \mathrm{e}^{-}\right.$) and excellent QE (one of the chips has a $\mathrm{QE}$ of $80 \%$ at $350 \mathrm{~nm})$.

\subsubsection{Fabry-Perot imaging spectroscopy}

Fabry-Perot (F-P) imaging spectroscopy (from 430-860 nm) forms one of the core capabilities of the RSS and will enable observations of both absorption and emission line sources of varying extent and at a variety of resolving powers: low $(\mathrm{R}=320-770)$, medium $(\mathrm{R}=1250-1650)$ and high $(\mathrm{R}=9000)$. The sensitivity to extended diffuse emission is afforded by the large collecting area of SALT, enabling the mapping of velocity structure and line strength and shape. The ability to both simultaneously detect and observe emission lines, for example in galaxy clusters or halos, is one advantage of the F-P mode. Coupling the polarimetric and F-P modes will provide a unique capability for imaging spectropolarimetry.

\subsubsection{Polarimetry modes}

The RSS polarimetric optics utilise a "wide-field" design (Nordsieck et al. 2003), employing a polarising beam-splitter consisting of a $3 \times 3$ array of calcite Wollaston prisms, deployable in the collimated beam. This takes the central half of the field and splits it into two orthogonally polarised fields corresponding to the ordinary $(\mathrm{O})$ and extraordinary $(\mathrm{E})$ beams. Polarisation modulation is done with a $1 / 2$ and/or $1 / 4$ waveplate, inserted just after the focal plane. By placing the beam splitter after the étalons, both the $\mathrm{E}$ and $\mathrm{O}$ fields have the same wavelength gradient in Fabry-Perot mode, enabling direct differencing of the two fields. One interesting characteristic of the Wollaston prisms is their intrinsic chromatic dispersion. This provides a very low resolution $(\mathrm{R} \sim 50)$ spectropolarimetric imaging mode (like objective prism spectroscopy) over the $4 \times 7.2$ (arcmin) ${ }^{2}$ field.

\subsection{SALTICAM imager}

SALTICAM was built at the SAAO, with one of us (DOD) as the Principal Investigator, and installed on SALT in June 2005. The instrument was conceived as a multi-purpose device, capable of performing roles as both an efficient acquisition camera and a scientific imager (Buckley et al. 2004b, O'Donoghue et al. 2003). SALTICAM was built in two stages, the first being installed in September 2003, when it served the purpose of the SALT Verification Instrument and was used to commission SALT and perform pointing, tracking, image quality and throughput tests.

In May 2005 SALTICAM was transformed to the so-called Acquisition Camera and Science Imager (ACSI) mode, in which focal reducing fore-optics were added. These provided focal reduction (from $\mathrm{f} / 4.2$ to $\mathrm{f} / 2$ ) giving a better (less oversampled) plate scale, allowing for the entire $\sim 10$ arcmin science and guidance fields to be imaged onto the $4 \mathrm{k} \times 4 \mathrm{k}$ detector mosaic.

The same frame transfer CCDs used in the RSS (O'Donoghue et al. 2004) allow for fast (at least $10 \mathrm{~Hz}$ ) acquisition, guiding and imaging. Together with the RSS, SALTICAM will extend the domain of time variability studies on $10 \mathrm{~m}$ class telescopes.

\section{SALT first science}

Scientific observations with SALT commenced in August 2005 with SALTICAM commissioning. One of the first programs attempted (O'Donoghue et al. 2006) was high time resolution photometry of eclipsing magnetic cataclysmic binary stars, also known as polars or AM Herculis stars. These objects emit the bulk of their luminosity through 

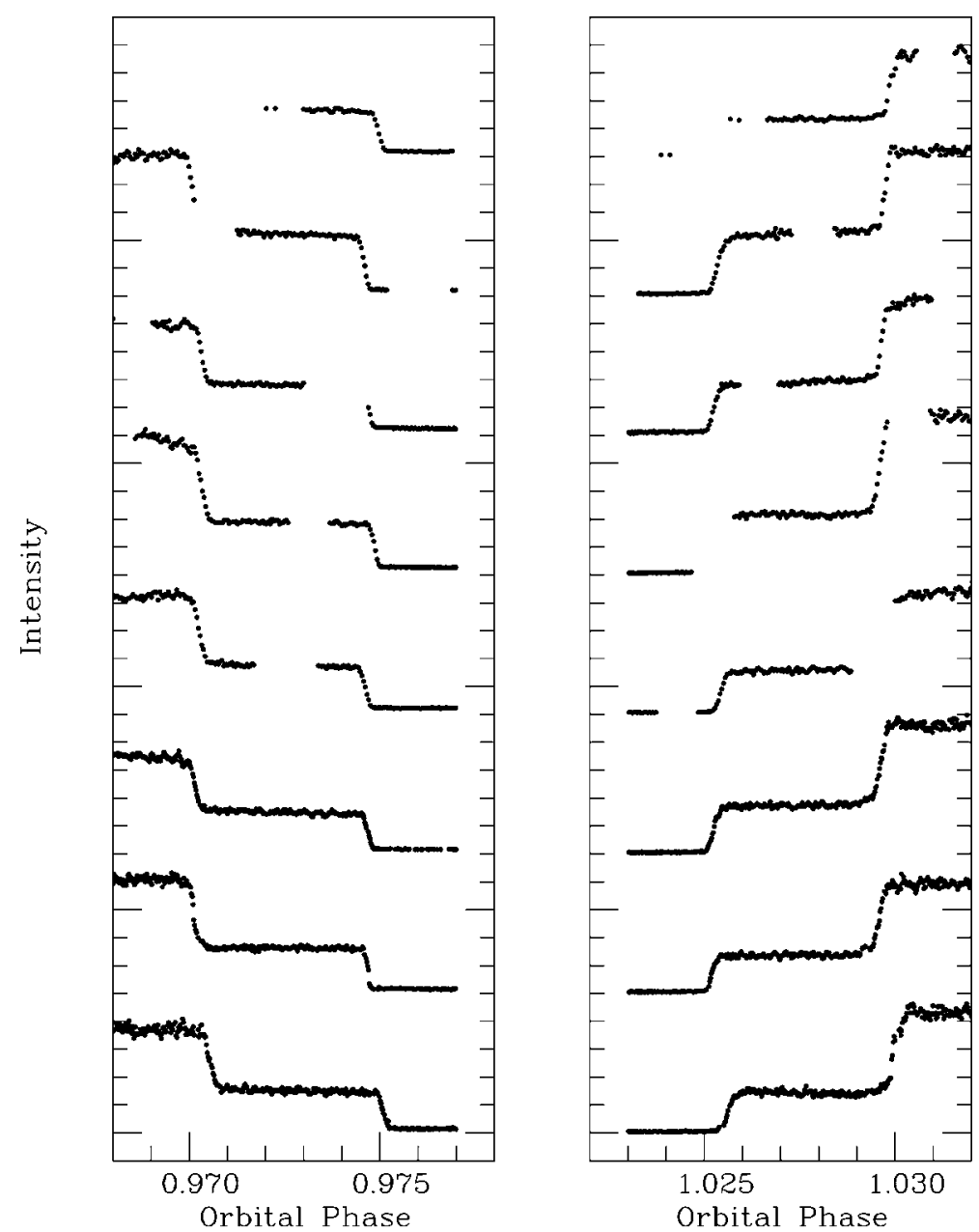

Figure 3. Light curves (white-light) of eclipse ingress (left) and egress (right) of the eclipsing polar SDSS015543+002807 taken by SALTICAM with 0.1 to $0.3 \mathrm{~s}$ resolution. The two steps are due to the successive disappearance and reappearance of the two accreting magnetic poles.

magnetic accretion onto a white dwarf. Hot spots on the surface emit in the UV-visible region through reprocessing of X-ray bremsstrahlung and optical-IR cyclotron radiation in the white dwarf photosphere and are located at, or near, the magnetic poles. High time resolution (ranging from 112 to $285 \mathrm{~ms}$ ) eclipse curves of unprecedented quality were obtained for one such system, SDSS015543+002807, a recently discovered deeply eclipsing $(V \sim 20$ at minimum) system, with a $1.45 \mathrm{~h}$ orbital period. In Fig. 3 we present a montage of the eclipse curves obtained in August and September 2005. These data clearly demonstrate that the bulk of the luminosity ( 95\%) comes from two hot-spots, located close to both magnetic poles, while the remaining light comes from the white dwarf photosphere and the accretion stream. The ingress/egress light-curves resolve the hot spots themselves, and have been used to determine their dimensions and extent.

This first science program was chosen partly to exercise the high-speed photometry mode of SALTICAM, but also because the observations were relatively easy to do, even without closed-loop guidance being implemented. The relative brightness of these polars 
( $V \sim 15-16$ outside eclipse) make them ideal commissioning targets, even in bright Moon, in poor seeing or for non-optimal image quality.

Other SALT science programs have also begun as part of "performance verification" $(\mathrm{P}-\mathrm{V})$ phase of the telescope, SALTICAM and RSS, which is expected to extend until May 2006. These programs have been accepted on a "best effort" basis and initially are aimed to be pilot programs or short observations with a potential quick and interesting scientific result, designed to exercise the different operational modes of the telescope and instruments. Given that many of the supporting facility instrumentation (e.g. for auto-guidance, auto-focus, atmospheric dispersion compensation, exit pupil baffling, calibrations, etc) is still undergoing installation and commissioning some P-V programs have had to be postponed. In addition, there is still ongoing telescope engineering work to improve image quality and reduce scattered light. All of this means that the telescope is not yet optimised for undertaking demanding observations of very faint objects. However, notwithstanding the above, scientifically useful observations are being conducted, including some reported in these proceedings (e.g. Fritze - v. Alvensleben et al. 2006).

\section{SALT lessons for ELT designs?}

Finally, in the context of this meeting, it is perhaps worth asking if there are any lessons learnt in building SALT that might have some relevance for future 300-100 m telescopes (e.g. Buckley et al. 2004a). Something which all telescope builders agree on is that the current cost curves, where typically the Cost proportional to (Diameter) ${ }^{2.5}$, needs to be significantly decreased if ELTs are to be affordable. This implies engineering innovations need to be applied to bring down costs, just as happened in the design of SALT and HET, where the costs are typically $\sim 20 \%$ of conventional design $8-10 \mathrm{~m}$ telescopes.

The major cost savings for the SALT/HET designs come from:

1.) Reducing the moving mass of the telescope,

2.) Fixing the tilt angle of the telescope, and

3.) Having identical (spherically figured) primary mirror segments.

These same principles could also be applied to ELT designs, as has been pointed out in the past (e.g. Sebring et al. 1998, Ray et al. 2000). The authors of those publications maintain that extrapolation of existing conventional 8-10 $\mathrm{m}$ telescope designs (e.g. VLT, Keck, Gemini) is insufficient and that engineering innovation is required. Some innovation is apparent in existing ELT designs, for example OWL, which is proposing a spherical primary mirror array and a complex spherical aberration corrector (Dierickx et al. 2004). However, the notion of fully steerable telescopes is still popular, despite the costs involved. While some science programs demand such an ability, many do not and it might still be prudent to consider options involving a fixed telescope/primary mirror. Wider investigations in design parameter space could well pay off and already suggestions have been made concerning potential extrapolations of the SALT SAC design for an ELT (O’Donoghue 2000).

\section{Conclusions}

The Southern African Large Telescope was completed in a rapid time frame of 5.5 years and within $1 \%$ of its original US $\$ 19.7 \mathrm{M}$ budget allocation. Two of its first generation instruments, the high-speed optical imager, SALTICAM, and the versatile prime focus imaging spectrograph, the Robert Stobie Spectrograph (RSS), are now installed and in various stages of commissioning. Early indications are that the telescope will meet its design goals and full scientific operations are expected in 2006. Already the first 
science paper based on SALTICAM observations has been completed and submitted for publication. The success of SALT is due to the design process, which followed a tailored, but rigorous, systems engineering approach and the skill, talent and hard work of the people comprising the SALT Project and Operations Teams, the support staff of the SAAO and the various instrument teams within the SALT consortium.

SALT has been an excellent example of how clever engineering solutions can solve demanding scientific requirements, but at a significantly reduced and, for a country like South Africa, affordable cost. While there were design compromises, SALT will nonetheless be a competitive facility with many unique modes. SALT is now beginning its scientific life and will provide quality data to the scientists within the partnership for many decades to come.

The financial constraints under which SALT was built meant that an innovative and cost effective design was required. Similar constraints exist for ELTs, and it is perhaps worth reappraising whether or not unconventional designs, like SALT and HET, and some compromises should be adopted in these much costlier and ambitious projects. The last thing the astronomical community needs is the prospect of a Texas Super-Conducting Super-Collider scenario!

\section{References}

Buckley, D.A.H., O’Donoghue, D., Sessions, N.J. \& Nordsieck, K.H. 2000, in: M. Iye \& A.F. Moorwood (eds.), SPIE 4008, 72

Buckley, D.A.H., Hearnshaw, J.B., Nordsieck, K.H. \& O'Donoghue, D. 2003, in: P. Guhathakurta (ed.), SPIE 4834, 264

Buckley, D.A.H., Meiring, J.G., Swiegers, J. \& Swart, G.P. 2004a, in: A. Ardeberg \& T. Andersen (eds.), SPIE 5382, 245

Buckley, D.A.H., Cottrell, P.L., Nordsieck, K.H., O'Donoghue, D. \& Williams, T.B. 2004b, in: A.F. Moorwood M. Iye (eds.), SPIE 5492, 60

Burgh, E.B., et al. 2003, in: M. Iye \& A.F. Moorwood (eds.), SPIE 4841, 1463

Dierickx, P., et al. 2004, in: J.M. Oschmann (ed.), SPIE 5489, 391

Fritze - v. Alvensleben, U., Papaderos, P., Anders, P., Lilly, M.T., Cunow, B. \& Gallagher, J. 2006, this volume

Kobulnicky et al. 2003, in: M. Iye \& A.F. Moorwood (eds.), SPIE 4841, 1634

Meiring, J.G., Buckley, D.A.H., Lomberg, M. \& Stobie, R.S. 2003, in: J.M. Oschmann \& L.M. Stepp (eds.), SPIE 4837, 11

Meiring, J.G. \& Buckley, D.A.H. 2004, in: J.M. Oschmann (ed.), SPIE 5489, 592

Nordsieck, K.H., Jaehnig, K.P., Burgh, E.B., Kobulnicky, H.A., Percival, J.W. \& Smith, M.P. 2003, in: S. Fineschi (ed.), SPIE 4843, 170

O'Donoghue, D. 2000, in: P. Dierickx (ed.), SPIE 4003, 363

O'Donoghue, D. \& Swat, A. 2001, in: R.G. Bingham \& D.D. Walker (eds.), SPIE 4411, 72

O'Donoghue, D., et al. 2003, in: M. Iye \& A.F. Moorwood (eds.), SPIE 4841, 465

O'Donoghue, D., et al. 2004, in: J.D. Garnett \& J.W. Beletic (eds.), SPIE 5499, 406

O'Donoghue, D., et al. 2006, MNRAS, submitted

Ramsey, L.W., et al. 1998, in L.M. Stepp (ed.), SPIE 3352, 34

Ray, F.B., Bash, F.N., Ramsey, L.W. \& Sebring, T. 2000, in: T.A. Sebring \& T. Andersen (eds.), SPIE 4004, 405

Sebring, T.A. \& Ramsey, L.W. 1997, in: A. L. Ardeberg (ed.), SPIE 2871, 32

Sebring, T.A., Bash, F.N., Ray, F.B. \& Ramsey, L.W. 1998, SPIE 3352, 792

Stobie, R.S., Meiring, J.G. \& Buckley, D.A.H. 2000, in: P. Dierickx (ed.), SPIE 4003, 355

Swart, G.P. \& Meiring, J.G. 2003, in: J.M. Oschmann \& L.M. Stepp (eds.), SPIE 4837, 166

Swart, G.P. \& Buckley, D.A.H. 2004, in: S.C. Craig \& M.J. Cullum (eds.), SPIE 5497, 62 


\section{Discussion}

CRABTREE: What are the foreseen operational costs of SALT?

BUCKLEY: The current estimate for 10 years operations for SALT is R80.43M (US\$12 M) including a 10 percent contingency.

IYE: Do you have a plan to implement AO based instruments? What would be the specific features in designing AO for SALT type telescope?

BUCKLEY: AO options as a future development for SALT have certainly been discussed. Phasing of the primary would require a phasing camera, but the current hardware (i.e. edge sensors and actuators) appears to have sufficient resolution ( $\sim 50 \mathrm{~nm} \mathrm{rms})$ to keep the mirror array phased. The moving pupil presents somewhat of a complication and would require significant design effort for a deformable mirror. Also the Prime Focus Payload puts limitations on mass and volume.

Hough: Has the expected instrumental polarisation been modelled for SALT?

BuCKLEY: Yes, Ken Nordsieck (PI for the Robert Stobie Spectrograph) has modelled the instrumental polarisation of the telescope, which is $<0.2 \%$ for all field angles and $<0.1 \%$ for field angles $<2$ arcmin. We expect to be able to reliably calibrate the polarimetric data to better than \pm 0.04 percent.

ZinneCKeR: You discussed how SALT learnt from HET. Did HET learn from SALT?

BUCKLEY: Yes. HET followed SALT's lead in implementing a natural ventilation system using louvers. Like SALT they have also installed a distance measuring interferometer and autocollimator on the Prime Focus Payload. They are also looking at the possibility of redesigning their Spherical Aberration Corrector.

DENNEFELD: The specific design of SALT must have some consequences on the available field of view. What are the constraints on a wide-field, fibre fed spectrograph, in terms of achievable view and possible changes/deformation (distortion) during the exposure?

BUCKLEY: SALT has an 8 arcmin diameter science field and a flat focal plane, but the telecentric angle increases to $\sim 5^{\circ}$ at the edge of the field. Pupil variations have little perceptible effect on the near field PSF, while azimuthal scrambling in the fibre efficiently removes all asymmetric structure in the far field. During an exposure the PSF remains stable while the far field pattern change can cause small focal ratio degradation changes. Experience on HET with their fibre fed High Resolution Spectrograph is that with calibration (using an iodine cell), radial velocity accuracies of a few $\mathrm{ms}^{-1}$ are achievable. 